\title{
DOMAIN RIBBONS INSIDE DOMAIN WALLS AT FINITE TEMPERATURE
}

\author{
F. A. Brito and D. Bazeia \\ Departamento de Física, Universidade Federal da Paraíba \\ Caixa Postal 5008, 58051-970 João Pessoa, Paraíba, Brazil
}

\begin{abstract}
In this paper we deal with defects inside defects in systems of two scalar fields in $3+1$ dimensions. The systems we consider are defined by potentials containing two real scalar fields, and so we are going to investigate domain ribbons inside domain walls. After introducing some general comments on the possibility of finding defects that support internal structure in two specific systems, we introduce thermal effects to show how the picture for domain walls hosting domain ribbons appears at high temperature.
\end{abstract}

PACS numbers: 11.10.Lm, 11.27.+d, 98.80.Cq 


\section{Introduction}

The possibility that the early universe may have experienced symmetry breaking phase transitions resulting in the formation of defects has provided a motivation for studies of several possible defect configurations - see for instance Ref. [1]. In this route to defect formation we can single out the case that considers the presence of defects inside defects. This possibility was initiated in [2], firstly within the context of superconducting strings, where one

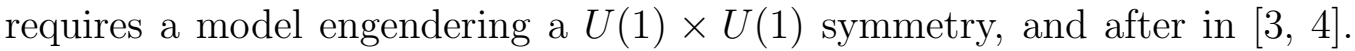
Other more recent works on the same issue can be found in [5, 6, 0, 8].

To implement the idea of finding defect inside defect, in general one considers systems of two scalar fields, in which the first field plays the usual role one finds in the standard route to defect formation, and the second field enters the game via a potential that couples it to the first field, in a way such that the system now allows for the presence of defects inside the defect originated by the first scalar field. This idea is usually implemented by introducing a general potential, depending on the two scalar fields and containing several parameters that are a posteriori tuned to allow for the presence of defects inside defects. Despite this general picture, it was recently shown in [8] that models belonging to a class of systems of two real scalar fields [9, 10, 11] also appear suitable to develop the idea related to internal structure of topological defects. In this case the model is controlled by a reduced number of parameters, and this may guide us toward a clearer understanding of some physical aspects of the system.

The systems we shall investigate are defined with two real scalar fields in $3+1$ dimensions, and present $Z_{2} \times Z_{2}$ symmetry that implements spontaneous symmetry breaking in the two independent field directions. Thus we shall be dealing with the presence of domain ribbons inside domain walls. Although domain walls may conflict with observations, because of wall domination within cosmological scenarios, there exist mechanisms that allow an avoidance of wall domination. One such mechanism relies on allowing the exact discrete symmetry to be replaced by an approximate discrete symmetry, and this can occurs for example as a consequence of supersymmetry breaking in supersymmetric theories [12]. When there are no fermions present, then the regions of higher energy density tend to shrink, allowing closed domain walls bubbles to colapse away. The presence of domain ribbons within walls is not expected to qualitatively change this process, although there may be 
a release of boson radiation from the ribbon.

When fermions are present, however, the situation may be a little more complicated. It has been pointed out [13] (within the context of a model containing no domain ribbons) that if fermions are coupled strongly to a domain wall field, and if the fermions, which are massive in the vacuum, become massless within the domain wall, then the Fermi gas within the wall can contribute a degeneracy pressure which tends to stabilize the surface area of the wall. However, the vacuum bag can flatten and fragment, ultimately producing tiny fermionically stabilized bags of false vacuum called "Fermi balls". The domain walls in this type of model can ultimately be replaced by Fermi balls, which can be cosmologically acceptable. It has been argued that this type of model can be obtained from a supersymmetric domain wall model [12], where supersymmetry breaking terms cause the exact wall-producing discrete symmetry to be replaced by an approximate one.

Consider now a system accommodating fermions and domain ribbons. A specific model is the supersymmetric system already investigated in Ref. [14]. In this model the fermions become massless inside the ribbons, but are massive outside the ribbons - both inside the domain wall and in the vacuum. Therefore there is a strong force attracting the fermions into the ribbons from the domain wall. Thus, fermions that are initially present within the wall may quickly be absorbed into the ribbons. A Fermi gas of massless fermions develops within a ribbon, allowing a closed ribbon loop to stabilize inside the wall.

Now let us again suppose that a small energy difference in the initially degenerate vacuum states develops, so that the exact discrete symmetry giving rise to the domain walls is replaced by an approximate discrete symmetry. As before, we expect the space to fill with closed bags of false vacuum, which tend to collapse. There are now two possibilities: (i) either the typical vacuum bag will collapse away before any ribbons form within it, or (ii) ribbons will form before the bag collapses completely. In case (i), the end result may be the production of stable Fermi balls. In case (ii), closed ribbon loops form within the typical vacuum bag, and the ribbons tend to absorb the fermions from the wall, so that a stable ribbon loop can reside in the vacuum bag. The above scenario serves to demonstrate the possible importance of finite temperature effects, since the dynamical pathways and intermediate states involved in cases (i) and (ii) leading to the possible end states may depend strongly on the difference between the critical temperatures for domain wall 
and domain ribbon formation.

To explore some of the above issues, in this work we shall firstly deal with classical features of the system introduced in [8] and of another system, presented in [6]. This last system is defined by a potential that is usually considered to develop the idea of introducing internal structure to topological defects, and so we shall be also offering a comparison between the standard procedure [6] and the alternative approach introduced in [8]. Owing to direct interest to cosmology, we shall calculate the effective potential, from which we obtain the high temperature effects in each one of these systems. The thermal effects are obtained by following the standard works [15, 16, 17], and here we remark that the above systems are defined by potentials that depend on two fields, and so the effective potential or, better, the thermal effects in general introduce two critical temperatures, driving symmetry breaking in each one of the two independent field directions. These investigations are organized as follows. In the next Section we introduce general considerations concerning the presence of domain ribbon inside domain wall in two specific systems. We also investigate classical or linear stability of the solutions we need to implement the idea of introducing defects inside defects. In Sec. 3 we calculate the effective potential and present the high temperature corrections to the classical potential. Here we obtain explicit expressions for the critical temperatures in each one of the two systems under consideration. We end the work in Sec. 目, where we comment on conclusions and possible generalizations of the present investigations.

\section{General Considerations}

We are interested in systems of two real scalar fields. In this case the general Lagrangian density is given by

$$
\mathcal{L}=\frac{1}{2} \partial_{\alpha} \phi \partial^{\alpha} \phi+\frac{1}{2} \partial_{\alpha} \chi \partial^{\alpha} \chi-U(\phi, \chi) .
$$

Here we are using natural units, in which $\hbar=c=1$, and the metric tensor $g^{\alpha \beta}$ is diagonal, with elements $[1,-1,-1,-1] . U=U(\phi, \chi)$ is the potential, in general a nonlinear function of the two fields. In the following we shall comment on some systems of two coupled real scalar fields described via the above Lagrangian density. 


\section{$2.1 \quad$ A Class of Systems}

The class of systems of two real scalar fields that we are interested in is defined by the following potential, as it was already stressed in [9, 10, 11],

$$
U(\phi, \chi)=\frac{1}{2} H_{\phi}^{2}+\frac{1}{2} H_{\chi}^{2}
$$

where $H=H(\phi, \chi)$ is a smooth but otherwise arbitrary function of the fields $\phi$ and $\chi$, and $H_{\phi}=\partial H / \partial \phi, H_{\chi}=\partial H / \partial \chi$. In this case, systems defined by the function $H(\phi, \chi)$ present some general and very interesting properties, mainly in $1+1$ dimensions. For instance, the second-order equations of motion for static solutions

$$
\begin{aligned}
& \frac{d^{2} \phi}{d x^{2}}=H_{\phi} H_{\phi \chi}+H_{\chi} H_{\phi \chi} \\
& \frac{d^{2} \chi}{d x^{2}}=H_{\phi} H_{\phi \chi}+H_{\chi} H_{\chi \chi}
\end{aligned}
$$

are solved by field configurations satisfying the following set of first-order differential equations

$$
\begin{aligned}
& \frac{d \phi}{d x}=H_{\phi}, \\
& \frac{d \chi}{d x}=H_{\chi} .
\end{aligned}
$$

The energy is bounded from bellow, and for configurations obeying the above first-order equations the energy gets to its minimum value, given by

$$
E_{B}=H(\phi(\infty), \chi(\infty))-H(\phi(-\infty), \chi(-\infty))
$$

Furthermore, the set of first order differential equations can be seen as a dynamical systems, and we can take advantage of all the mathematical tools available to dynamical systems to deal with those equations. In particular, one sees that the singular points of the corresponding dynamical system are all the possible minimum energy states of the field system, and so they are identified to the true vacuum states of the system. On the other hand, all static configurations we can find in the above class of systems are classicaly or linearly stable. This is interesting, and shows that perturbative quantum corrections about static configurations can be done by just following the standard procedure - see, for instance, Ref. [18]. 


\section{$2.2 \quad$ First System of Two Fields}

As a first example, let us focus attention on the system defined by

$$
H(\phi, \chi)=\lambda\left(\frac{1}{3} \phi^{3}-a^{2} \phi\right)+\mu \phi \chi^{2} .
$$

In this case the potential is given by

$$
U(\phi, \chi)=\frac{1}{2} \lambda^{2}\left(\phi^{2}-a^{2}\right)^{2}+\lambda \mu\left(\phi^{2}-a^{2}\right) \chi^{2}+2 \mu^{2} \phi^{2} \chi^{2}+\frac{1}{2} \mu^{2} \chi^{4} .
$$

This is the system already investigated in [8], and here we return to it to show that it engenders some very specific features, unrealized in Ref. [8]. To see this, let us first search for the vacuum states: They are four, two at $\chi=0$ and $\phi_{0}^{2}=a^{2}$, and two at $\phi=0$ and $\chi_{0}^{2}=r a^{2}$. For simplicity, here we are using $\lambda=\mu r$, and $r$ is a real, positive and dimensionless parameter.

The potential presents the following tipical forms

$$
\begin{aligned}
U(\phi, 0) & =\frac{1}{2} \mu^{2} r^{2}\left(\phi^{2}-a^{2}\right)^{2}, \\
U(0, \chi) & =\frac{1}{2} \mu^{2}\left(\chi^{2}-r a^{2}\right)^{2} .
\end{aligned}
$$

In this case we see that both $U(\phi, 0)$ and $U(0, \chi)$ present spontaneous symmetry breaking, and this is all we need for building defects inside defects in the above system. In this case we can introduce meson masses

$$
\begin{aligned}
& m_{\phi}^{2}\left(\phi_{0}^{2}, 0\right)=4 \mu^{2} r^{2} a^{2}, \\
& m_{\chi}^{2}\left(0, \chi_{0}^{2}\right)=4 \mu^{2} r a^{2}
\end{aligned}
$$

and so $m_{\phi}^{2}\left(\phi_{0}^{2}, 0\right)=r m_{\chi}^{2}\left(0, \chi_{0}^{2}\right)$. On the other hand, the potential also gives

$$
\begin{aligned}
U\left(\phi_{0}^{2}, \chi\right) & =2 \mu^{2} a^{2} \chi^{2}+\frac{1}{2} \mu^{2} \chi^{4}, \\
U\left(\phi, \chi_{0}^{2}\right) & =2 \mu^{2} r a^{2} \phi^{2}+\frac{1}{2} \mu^{2} r^{2} \phi^{4} .
\end{aligned}
$$

Here we can also introduce meson masses

$$
\begin{aligned}
& m_{\phi}^{2}\left(\phi, \chi_{0}^{2}\right)=4 \mu^{2} r a^{2}, \\
& m_{\chi}^{2}\left(\phi_{0}^{2}, \chi\right)=4 \mu^{2} a^{2},
\end{aligned}
$$


and now $m_{\phi}^{2}\left(\phi, \chi_{0}^{2}\right)=r m_{\chi}^{2}\left(a^{2}, \chi\right)$. We also have $m_{\phi}^{2}\left(\phi_{0}^{2}, 0\right)=r m_{\phi}^{2}\left(\phi, r a^{2}\right)$ and $m_{\chi}^{2}\left(0, \chi_{0}^{2}\right)=r m_{\chi}^{2}\left(\phi_{0}^{2}, \chi\right)$. The parameter $r$ controls the meson masses, and we see that for $r=1$ (that is $\lambda=\mu$ ) the above mass values degenerate to the single value $4 \mu^{2} a^{2}$.

At this point we realize that for $r \neq 1$, that is, for $\lambda \neq \mu$, the system presents discrete $Z_{2} \times Z_{2}$ symmetry. The limit $r \rightarrow 1$ introduces the $Z_{4}$ symmetry, and this means that the two fields have the same physical significance. This seems to pose the question of whether will the system choose the field to host the other field, to lead to defect inside defect. However, a closer investigation shows that this question is in fact nonsense since the limit $r \rightarrow 1$ should be avoided, because in this case the system of two coupled fields degenerate into two systems of a single field each one. To see how this works explicitly, let us rotate the $(\phi, \chi)$ plane to the $\left(\phi_{+}, \phi_{-}\right)$plane, where $\phi_{ \pm}=2^{-1 / 2}(\chi \pm \phi)$. In this case $H$ can be cast to the form

$$
H\left(\phi_{+}, \phi_{-}\right)=2^{-1 / 2} \mu\left[F_{r}\left(\phi_{+}, \phi_{-}\right)-F_{r}\left(\phi_{-}, \phi_{+}\right)\right],
$$

where the function $F$ is given by

$$
F_{r}\left(\phi_{ \pm}, \phi_{\mp}\right)=\frac{1}{2}\left(1+\frac{1}{3} r\right) \phi_{ \pm}^{3}-r a^{2} \phi_{ \pm}+\frac{1}{2}(1-r) \phi_{ \pm}^{2} \phi_{\mp} .
$$

Here we see that the limit $r \rightarrow 1$ decouples $\phi_{+}$from $\phi_{-}$, and so there is no interaction between the two fields. A lesson to learn is then that although the original system has two independent parameters, namely $\lambda$ and $\mu$, only their ratio $\lambda / \mu$ or $r$ is physically relevant to the issues under consideration, and this ratio should only take values in each one of the two distinct regions $r \in(0,1)$ or $r \in(1, \infty)$.

Let us now focus attention on defect formation. We see that the potential $U(\phi, 0)$ presents spontaneous symmetry breaking, and so we can have the kink solution

$$
\phi(x)=a \tanh (\mu r a x),
$$

with energy $E_{\phi}=(4 / 3) \mu r a^{3}$. However, from $U(0, \chi)$ we also have the kink solution

$$
\chi(y)=a r^{1 / 2} \tanh \left(\mu r^{1 / 2} a y\right),
$$

with energy $E_{\chi}=(4 / 3) \mu r^{3 / 2} a^{3}$. Here we have $E_{\chi}=r^{1 / 2} E_{\phi}$, and so the parameter $r$ also controls the energy ratio for defect formation. The picture 
is then the following: The domain wall generated by the kink of one of the two fields will host the domain ribbon generated by the kink of the other field; the host and the nested fields are determined by the value of the single parameter $r \neq 1$, which is the same parameter that controls how mesons of the nested field prefer to live inside or outside the domain wall.

\subsection{Second System of Two Fields}

As a second example, let us now consider the potential

$$
\begin{aligned}
V(\phi, \chi)= & \frac{1}{2} \mu^{2} r^{2}\left(\phi^{2}-a^{2}\right)^{2}+\mu^{2}\left(\phi^{2}-a^{2}\right) \chi^{2}+ \\
& +\mu^{2} a^{2} b^{2} \chi^{2}+\frac{1}{2} \mu^{2} c^{2} \chi^{4} .
\end{aligned}
$$

Here $r, b$, and $c$ are real and positive parameters, and now the system is of the form considered in [6]. This potential presents the following tipical forms

$$
\begin{aligned}
V(\phi, 0) & =\frac{1}{2} \mu^{2} r^{2}\left(\phi^{2}-a^{2}\right)^{2} \\
V(0, \chi) & =\frac{1}{2} \mu^{2} r^{2} a^{4}-\mu^{2} a^{2}\left(1-b^{2}\right) \chi^{2}+\frac{1}{2} \mu^{2} c^{2} \chi^{4} .
\end{aligned}
$$

We shall assume that $0<b^{2}<1$. In this case we see that both $V(\phi, 0)$ and $V(0, \chi)$ present spontaneous symmetry breaking. However, while the values $\phi_{0}^{2}=a^{2}$ and $\chi=0$ are true vacuum states, the values $\phi=0$ and $\chi_{0}^{2}=\left[\left(1-b^{2}\right) / c^{2}\right] a^{2}$ are just local minima of the potential. We make these local minima to be true vacuum states by reducing the number of independent parameter, requiring that $r^{2} c^{2}=\left(1-b^{2}\right)^{2}$. For simplicity we set $1-b^{2}=s^{2}$ and the potential is now written in terms of two parameters, namely $r \in$ $(0, \infty)$ and $s \in(0,1)$. In particular, $V(0, \chi)$ can be cast to the form

$$
V(0, \chi)=\frac{1}{2} \mu^{2} \frac{s^{4}}{r^{2}}\left(\chi^{2}-\frac{r^{2}}{s^{2}} a^{2}\right)^{2},
$$

and now there are true vacuum states also at $\phi=0$ and $\chi_{0}^{2}=\left(r^{2} / s^{2}\right) a^{2}$. Here we note that the potential $V(\phi, \chi)$, written in terms of these two parameters $r$ and $s$, does not reproduce the potential $U(\phi, \chi)$ of the former system anymore. Thus, this second system is different of the first system in the entire region of parameters $r \in(0, \infty)$ and $s \in(0,1)$. 
In this case we have the meson masses

$$
\begin{aligned}
& m_{\phi}^{2}\left(\phi_{0}^{2}, 0\right)=4 \mu^{2} r^{2} a^{2}, \\
& m_{\chi}^{2}\left(0, \chi_{0}^{2}\right)=4 \mu^{2} s^{2} a^{2},
\end{aligned}
$$

and so $m_{\phi}^{2}\left(\phi_{0}^{2}, 0\right)=\left(r^{2} / s^{2}\right) m_{\chi}^{2}\left(0, \chi_{0}^{2}\right)$. On the other hand, the potential also gives

$$
\begin{aligned}
& V\left(\phi, \chi_{0}^{2}\right)=\mu^{2} a^{2} \frac{r^{2}}{s^{2}}\left(1-s^{2}\right) \phi^{2}+\frac{1}{2} \mu^{2} r^{2} \phi^{4}, \\
& V\left(\phi_{0}^{2}, \chi\right)=\mu^{2} a^{2}\left(1-s^{2}\right) \chi^{2}+\frac{1}{2} \mu^{2} \frac{s^{4}}{r^{2}} \chi^{4},
\end{aligned}
$$

and we can also introduce meson masses

$$
\begin{aligned}
& m_{\phi}^{2}\left(\phi, \chi_{0}^{2}\right)=2 \mu^{2} \frac{r^{2}}{s^{2}}\left(1-s^{2}\right) a^{2}, \\
& m_{\chi}^{2}\left(\phi_{0}^{2}, \chi\right)=2 \mu^{2} a^{2}\left(1-s^{2}\right),
\end{aligned}
$$

and now $m_{\phi}^{2}\left(\phi, \chi_{0}^{2}\right)=\left(r^{2} / s^{2}\right) m_{\chi}^{2}\left(\phi_{0}^{2}, \chi\right)$. We also have $m_{\phi}^{2}\left(\phi_{0}^{2}, 0\right)=\left[2 s^{2} /(1-\right.$ $\left.\left.s^{2}\right)\right] m_{\phi}^{2}\left(\phi, \chi_{0}^{2}\right)$ and $m_{\chi}^{2}\left(0, \chi_{0}^{2}\right)=\left[2 s^{2} /\left(1-s^{2}\right)\right] m_{\chi}^{2}\left(\phi_{0}^{2}, \chi\right)$. Here we notice that $r$ and $s$ control the meson masses, and there are many possible choices for these parameters.

Let us now investigate defect formation. From the potential $V(\phi, 0)$ we can contruct the kink solution $\phi(x)=a \tanh (\mu \operatorname{rax})$, which has the same energy we have already calculated in the former system, namely $E_{\phi}=$ $(4 / 3) \mu r a^{3}$. In this case, however, from $V(0, \chi)$ we have

$$
\chi(y)=(r / s) a \tanh (\mu s a y),
$$

and the corresponding energy is $E_{\chi}=(4 / 3) \mu r(r / s) a^{3}$. Here we get $E_{\chi}=$ $(r / s) E_{\phi}$, and so we can control this energy relation by just controlling the ratio between the two parameters $r$ and $s$.

Here the picture is richer than the one that appears in the former system, evidently. For instance, from the above calculations we see that values at $s=r$ in the range $(0,1)$ are interesting values. Furthermore, the value $s^{2}=1 / 3$ is very peculiar and imposes no restriction on $r$ : This appears from the meson masses, which allow introducing the function

$$
g\left(s^{2}\right)=\frac{2 s^{2}}{1-s^{2}}
$$


This function depends only on $s^{2}$ and controls the ratio between meson masses of the field to be nested inside the domain wall. However, since $g\left(s^{2}\right) \leq 1$ for $s^{2} \leq 1 / 3$, and $g\left(s^{2}\right)>1$ for $s^{2}>1 / 3$, we see that evaporation of domain ribbons [6] into elementary mesons may or may not induce back reaction on the domain ribbon, and this appears to be controlled by the parameter $s$. As we have already shown, this is not the case in the former model since there we have just one parameter, and so there is no other parameter to be tuned anymore. For $s^{2}=1 / 3$ the above function becomes unit, and the meson masses degenerate into a single value, irrespective of the meson being inside or outside the domain wall.

\subsection{Classical Stability}

Since we are interested in implementing the idea of introducing internal structure to topological defects, we should also investigate if the topological defects are classically or linearly stable. Such a investigation seems to be important because it put forward results that may unveil the range of parameters where perturbative quantum corrections can be implemented standardly.

This is the main motivation to investigate classical stability of the pairs of solutions we have already introduced. Before doing that, however, we recall that the defects one is dealing with comes from kinks that appear in the corresponding $1+1$ dimensional systems, and so the informations we are requiring can be obtained by just investigating these $1+1$ systems. Furthermore, we already know [10, 11] that the first system presents stable solutions. Thus, we are left with the issue of investigating classical stability only for the second system.

This system is identified by the following potential

$$
V(\phi, \chi)=\frac{1}{2} \mu^{2} r^{2}\left(\phi^{2}-a^{2}\right)^{2}+\mu^{2} \phi^{2} \chi^{2}-\mu^{2} a^{2} s^{2} \chi^{2}+\frac{1}{2} \mu^{2} \frac{s^{4}}{r^{2}} \chi^{4} .
$$

As we have already shown, it presents the two pair of solutions:

$$
\begin{aligned}
& \phi_{1}(x)=a \tanh (\mu \operatorname{rax}), \quad \chi_{1}(x)=0, \\
& \chi_{2}(x)=(r / s) a \tanh (\mu \operatorname{sax}), \quad \phi_{2}(x)=0 .
\end{aligned}
$$

We consider fluctuations about each one of these two pair of solutions, in the form $\phi(x, t)=\phi(x)+\sum_{i} \eta_{i} \cos \left(w_{i} t\right)$ and $\chi(x, t)=\chi(x)+\sum_{i} \xi_{i} \cos \left(w_{i} t\right)$. We 
procced standardly, and we get the following Schrödinger operators, which respond for classical or linear stability,

$$
S_{d}^{(1,2)}=-\frac{d^{2}}{d x^{2}}+V_{d}^{(1,2)}(x)
$$

where $d=\phi \phi$ or $d=\chi \chi$, and

$$
\begin{aligned}
V_{\phi \phi}^{(1)}(x) & =4 \mu^{2} r^{2} a^{2}+6 \mu^{2} r^{2}\left(\phi_{1}^{2}-a^{2}\right) \\
V_{\chi \chi}^{(1)}(x) & =2 \mu^{2} a^{2}\left(1-s^{2}\right)+2 \mu^{2}\left(\phi_{1}^{2}-a^{2}\right) \\
V_{\phi \phi}^{(2)}(x) & =2 \mu^{2} \frac{r^{2}}{s^{2}} a^{2}\left(1-s^{2}\right)+2 \mu^{2}\left(\chi_{2}^{2}-\frac{r^{2}}{s^{2}} a^{2}\right) \\
V_{\chi \chi}^{(2)}(x) & =4 \mu^{2} s^{2} a^{2}+6 \mu^{2} \frac{s^{4}}{r^{2}}\left(\chi_{2}^{2}-\frac{r^{2}}{s^{2}} a^{2}\right) .
\end{aligned}
$$

The above problems were already solved in quantum mechanics. They are identified to modified Pöschl-Teller systems, and everything one needs is given in Ref. [19]. The general results can be resumed as follows: For the first pair of solutions, that connects $(-a, 0)$ to $(a, 0)$ by a straight line with $\chi=0$ we have to introduce the condition

$$
2 s^{4}+r^{2} s^{2} \leq r^{2}
$$

in order to ensure stability of this pair of solutions. For the second pair of solutions, that connects $(0,-(r / s) a)$ to $(0,(r / s) a)$ by a straight line with $\phi=0$ we have to introduce the condition

$$
2 r^{2}+s^{2} \leq 1
$$

These conditions appear after investigating the minimum energy eingenvalue of each one of the four Schrödinger operators just introduced.

The above results (42) and (43) show that there is room for choosing the parameters $r$ and $s$ without changing stability of the solutions. In particular, if one sets $s^{2}=1 / 3$, Eqs. (42) and (43) imply that $r^{2}=1 / 3$, also. Here we recall that the value $s^{2}=1 / 3$ was already shown to be peculiar, since it makes the field that generates defects to be nested inside the domain wall to have the same mass, irrespective of being inside or outside the wall. Furthermore, if one sets $r=s$, one sees from (42) and (43) that now one has 
stable solutions only in the range $r^{2}=s^{2} \in(0,1 / 3]$. Recall that $r=s$ makes the energy of each one of the two solutions we are considering to colapse into a single value. These results are interesting and will be further considered in the next Section, where we deal with high temperature effects.

\section{$3 \quad$ High Temperature Effects}

The above investigations lead us to pictures for building defects inside defects at zero temperature. However, to present investigations appropriate to the standard cosmological scenario we think that we should consider thermal effects, since one knows that the cosmic evolution occurs via expansion and cooling. Toward this goal, let us now deal with the effective potential, in order to investigate how the vacuum states of the system of two coupled real scalar fields change when the high temperature corrections are introduced.

In the following we shall first review the main steps to get to the thermal effects in the general system of two real scalar fields. In the sequel, we introduce the results for the specific class of systems of two scalar fields, defined via the function $H(\phi, \chi)$. Our investigation follows with two subsections, in which we calculate the critical temperatures for each one of the two systems introduced in the former Section.

\subsection{General Calculations}

We follow the standard route to symmetry breaking at high temperature, as we have already learned from the works [15, 16, 17]. In this case the one loop contributions to the effective potential can be cast to the general form

$$
U^{1}=\frac{1}{2} \int \frac{d^{\nu} k}{(2 \pi)^{\nu}} \ln \operatorname{det} M
$$

where the matrix $M$ is given by

$$
M=\left(\begin{array}{cc}
k^{2}+U_{\phi \phi} & U_{\phi \chi} \\
U_{\chi \phi} & k^{2}+U_{\chi \chi}
\end{array}\right),
$$

where the derivative of the potential has to be calculated at constant and uniform field configurations. We can rewrite this result as

$$
U^{1}=\frac{1}{2} \int \frac{d^{\nu} k}{(2 \pi)^{\nu}}\left[\ln \left(k^{2}+M_{+}^{2}\right)+\ln \left(k^{2}+M_{-}^{2}\right)\right],
$$


where

$$
M_{ \pm}^{2}=\frac{1}{2}\left(U_{\phi \phi}+U_{\chi \chi}\right) \pm \frac{1}{2} \sqrt{\left(U_{\phi \phi}+U_{\chi \chi}\right)^{2}-4 U_{\phi \chi} U_{\chi \phi}}
$$

To get to the thermal effects we should set

$$
\int d k_{0} \rightarrow \frac{1}{2 \beta} \sum_{n=-\infty}^{\infty}, \quad k_{0} \rightarrow \frac{2 n \pi}{\beta}, \quad \beta=\frac{1}{T}
$$

In this case we have

$$
U_{\beta}^{1}=\frac{1}{2 \beta} \sum_{i} \sum_{n=-\infty}^{\infty} \int \frac{d^{\nu-1} k}{(2 \pi)^{\nu-1}} \ln \left(\frac{4 \pi^{2} n^{2}}{\beta^{2}}+E_{M_{i}}^{2}\right),
$$

where we have set $E_{M_{i}}^{2}=\vec{k}^{2}+M_{i}^{2}$, with the understanding that $M_{1}=M_{+}$ and $M_{2}=M_{-}$.

Let us now work in the $3+1$ dimensional spacetime. In this case, after performing summation and integration we get, taking into account only the high temperature effects,

$$
U_{\beta}^{1}=\frac{1}{24 \beta^{2}}\left(M_{+}^{2}+M_{-}^{2}\right) .
$$

We use the values presented in Eq. (47) to obtain

$$
U_{\beta}^{1}=\frac{1}{24 \beta^{2}}\left(U_{\phi \phi}+U_{\chi \chi}\right),
$$

and so we get to the final result

$$
U_{\beta}=U(\phi, \chi)+\frac{1}{24 \beta^{2}}\left(U_{\phi \phi}+U_{\chi \chi}\right) .
$$

Symmetry exists when there is no spontaneous symmetry breaking. However, since we are dealing with two fields we have to impose conditions for the two independent field directions, and this will lead us to two critical temperatures. Here the results are

$$
\begin{aligned}
\left(T_{\phi}^{c}\right)^{2} & =-24 \frac{\bar{U}_{\phi \phi}}{\bar{U}_{\phi \phi \phi \phi}+\bar{U}_{\chi \chi \phi \phi}} \\
\left(T_{\chi}^{c}\right)^{2} & =-24 \frac{\bar{U}_{\chi \chi}}{\bar{U}_{\phi \phi \chi \chi}+\bar{U}_{\chi \chi \chi \chi}}
\end{aligned}
$$


where the bar over the potential indicates that after derivating the potential we should set $\phi=0$ and $\chi=0$.

For systems defined by $H(\phi, \chi)$, the above expressions for the critical temperatures can be written in a better form, in terms of the function $H$. In this case we have to replace, for instance,

$$
\begin{aligned}
& U_{\phi \phi} \rightarrow H_{\phi \phi}^{2}+H_{\phi} H_{\phi \phi \phi}+H_{\phi \chi}^{2}+H_{\chi} H_{\phi \phi \chi} \\
& U_{\chi \chi} \rightarrow H_{\chi \chi}^{2}+H_{\chi} H_{\chi \chi \chi}+H_{\phi \chi}^{2}+H_{\phi} H_{\phi \chi \chi} .
\end{aligned}
$$

The other terms can be written straightforwardly. However, if one takes the point of view that perhaps the most interesting systems are defined by potentials that contain at most the fourth power in the fields, then we should only consider functions $H(\phi, \chi)$ that contain at most third power in the fields. In this case we have simpler expressions for the quartic derivative of the potential, and they are, explicitly,

$$
\begin{aligned}
U_{\phi \phi \phi \phi} \rightarrow & 3\left(H_{\phi \phi \phi}^{2}+H_{\phi \phi \chi}^{2}\right), \\
U_{\chi \chi \chi \chi} \rightarrow & 3\left(H_{\chi \chi \chi}^{2}+H_{\phi \chi \chi}^{2}\right), \\
U_{\phi \phi \chi \chi} \rightarrow & 2\left(H_{\phi \phi \chi}^{2}+H_{\phi \chi \chi}^{2}\right)+ \\
& +H_{\phi \phi \phi} H_{\phi \chi \chi}+H_{\chi \chi \chi} H_{\phi \phi \chi} .
\end{aligned}
$$

The above results are general results, and now we focus attention on the two systems already introduced in Sec. 2 to calculate explicit expressions for their critical temperature.

\subsection{Critical Temperature in the First System}

We are interested in investigating the system defined via the function $H(\phi, \chi)$ introduced in Eq. (8). Here the critical temperatures are given by

$$
\begin{aligned}
\left(T_{\phi}^{c}\right)^{2} & =\frac{12 r^{2} a^{2}}{3 r^{2}+r+2} \\
\left(T_{\chi}^{c}\right)^{2} & =\frac{12 r^{2} a^{2}}{r(r+5)}
\end{aligned}
$$

These results show that

$$
\left(T_{\phi}^{c}\right)^{2}=\left(T_{\chi}^{c}\right)^{2} f(r)
$$


where $f(r)$ is given by

$$
f(r)=\frac{r(r+5)}{3 r^{2}+r+2} .
$$

We see that $f(0)=0, f(1)=1$, and $f(\infty)=1 / 3$. Furthermore, $f(r)$ is monotonicaly increasing for $r \in(0,1)$, and monotonicaly decreasing for $r \in(1, \infty)$, with $f(1)=1$ as its maximun value. This leads to the result that $\left(T_{\phi}^{c}\right)^{2}$ is always lesser than $\left(T_{\chi}^{c}\right)^{2}$, irrespective of the value of $r$. We remark that $T_{\phi}^{c}$ and $T_{\chi}^{c}$ cannot coincide because $r \neq 1$.

\subsection{Critical Temperature in the Second System}

Let us now focus attention on the second system, defined via the potential given by Eq. (34). Here the critical temperatures are given by

$$
\begin{aligned}
& \left(\bar{T}_{\phi}^{c}\right)^{2}=\frac{12 r^{2} a^{2}}{1+3 r^{2}}, \\
& \left(\bar{T}_{\chi}^{c}\right)^{2}=\frac{12 r^{2} s^{2} a^{2}}{r^{2}+3 s^{4}} .
\end{aligned}
$$

We can write

$$
\left(\bar{T}_{\phi}^{c}\right)^{2}=\left(\bar{T}_{\chi}^{c}\right)^{2} f(r, s)
$$

where the function $f(r, s)$ is given by

$$
f(r, s)=\frac{1}{1+3 r^{2}}\left(\frac{r^{2}}{s^{2}}+3 s^{2}\right) .
$$

This function presents the following two interesting possibilities of being unit: For $r=s$, in the interval $(0,1)$, and for $s^{2}=1 / 3$, irrespective of the value of $r$. However, from stability results of the former Section we see that the two critical temperatures may colaspe into a single one in the range $r^{2}=s^{2} \in(0,1 / 3]$.

\subsection{High Temperature Considerations}

The high temperature results obtained for the first system show that such system breaks the symmetry firstly in one field direction, and it is only after this symmetry breaking that the second symmetry breaking will appear. On 
the other hand, we know that this system allows the presence of defects inside defects only after the occurrence of the second symmetry breaking. These critical temperatures are $T_{\chi}^{c}$ and $T_{\phi}^{c}$, respectively, and so we see that there is a temperature range, that can be written via $t=T / T_{\chi}^{c}$ as $\sqrt{f(r)} \leq t \leq 1$, in which the system only supports structureless domain walls. In the second system there is a range in parameter space where $r^{2}=s^{2} \in(0,1 / 3]$ that makes the two temperatures $\bar{T}_{\phi}^{c}$ and $\bar{T}_{\chi}^{c}$ to colapse into a single one.

Another interesting issue concerns structureless domain walls versus domain walls that support domain ribbons. To shed some light on this, let us first recall that $U(\phi, \chi)$ given by (9) has the following single field limits

$$
\begin{aligned}
U(\phi, 0) & =\frac{1}{2} \mu^{2} r^{2}\left(\phi^{2}-a^{2}\right)^{2}, \\
U(0, \chi) & =\frac{1}{2} \mu^{2}\left(\chi^{2}-r a^{2}\right)^{2} .
\end{aligned}
$$

However, from the results for the critical temperature in the first model we see that $\left(T_{\chi}^{c}\right)^{2}$ is always greater than $\left(T_{\phi}^{c}\right)^{2}$. Thus if one thinks on defect formation within the cosmological scenario, it is not unreasonable to suppose that the host domain wall is generated by the $\chi$ field. Owing to compare this to structureless domain walls we focus attention on the (single field) system defined by $U(0, \chi)$.

We recall that $r$ is real and positive, and so we see that the above potential $U(0, \chi)$ presents standard domain wall, structureless. For this system the critical temperature can be written as

$$
T_{c}^{2}=4 r a^{2}
$$

and so we can get

$$
\left(T_{\chi}^{c}\right)^{2}=\left(\frac{3}{5+r}\right) T_{c}^{2}
$$

The above result compares the critical temperature for formation of structureless domain walls in a system of just one field to the critical temperature for formation of domain walls that support domain ribbons in the first system of two fields. This result shows that the presence of the second field, which responds for nesting defects inside the wall, contributes reducing the critical temperature. The reduction depends on $r$, and this parameter can be controlled to directly affect the picture for defect formation within the cosmological scenario. 
Another high temperature result can be introduced in the following way: In the first system of two fields we still consider $r$ real and positive, but now we make $\lambda=-\mu r$. This possibility was already considered in [20], but there the motivation is directly related to investigations of an enlarged system where the symmetry $Z_{2} \times Z_{2}$ is changed to become $Z_{2} \times U(1)$, with $U(1)$ implemented globally. The present interest is however to keep the symmetry as the discrete $Z_{2} \times Z_{2}$. In this case the potential $U(\phi, \chi)$ given by (9) changes to

$$
\bar{U}(\phi, \chi)=\frac{1}{2} \mu^{2} r^{2}\left(\phi^{2}-a^{2}\right)^{2}-\mu^{2} r\left(\phi^{2}-a^{2}\right) \chi^{2}+2 \mu^{2} \phi^{2} \chi^{2}+\frac{1}{2} \mu^{2} \chi^{4} .
$$

Now it is not hard to realize that spontaneous symmetry breaking gets implemented only from the $Z_{2}$ symmetry associated to the $\phi$ field. This means that domain walls generated by this $\phi$ field cannot host domain ribbons. Although in this new model domain ribbons cannot be nested inside domain walls anymore, we believe that it is still interesting to investigate how the high temperature effects enter the game in this case too. Here we take advantage of the investigations already done to introduce the ratio between the critical temperatures in this system and in the (single field) system defined by $\bar{U}(\phi, 0)$. This ratio is controlled by $\left[3 r^{2} /\left(3 r^{2}-r+2\right)\right]^{1 / 2}$. It is lesser or equal to unit for $r \in(0,2]$, and greater for $r \in(2, \infty)$, and so the critical temperature in the system of two fields defined by $\bar{U}(\phi, \chi)$ is lesser or equal to the critical temperature in the system of one field defined by $\bar{U}(\phi, 0)$ for $r$ lesser or equal to 2 , and greater for $r$ greater than 2 .

\section{Comments and Conclusions}

In this work we have investigated the possibility of introducing defects inside defects in systems of two real scalar fields. After presenting some general considerations, we have investigated the high temperature thermal effects to the classical potential. These investigations were done on two specific systems, the first being defined by a function $H=H(\phi, \chi)$, and the other defined by a more general potential, as occurs in models usually considered to build defects inside defects. The basic motivation for investigating these two systems is to provide a comparison between the standard approach to defects inside defects, and the alternative route recently introduced in [8]. 
The present investigations show that systems belonging to a general class of systems of two real scalar fields present all the features one needs to implement the idea of nesting domain ribbons inside domain walls. These systems are simpler because they are defined via the function $H(\phi, \chi)$, are controlled by a reduced set of parameters, present stable configurations, and can be extended to become supersymmetric [20] very naturally. This is interesting since one keeps the underlying features of systems clearer than the features that appear in the standard approach. As we have seen, however, in this system there are two critical temperatures, driving symmetry breaking in each one of the two independent field directions. The possibility of having two distinct critical temperatures implies that the internal structure of the domain wall cannot appear simultaneously with the domain wall itself. This is in distinction to results in the second system, in which it is possible to introduce a single critical temperature to drive symmetry breaking in the two field directions simultaneously. The relation between the two critical temperatures in the first system is $\left(T_{\phi}^{c}\right)^{2}=\left[r(r+5) /\left(3 r^{2}+r+2\right)\right]\left(T_{\chi}^{c}\right)^{2}$ and depends on $r$, the ratio between the two parameters $\lambda$ and $\mu$ that defines the system. In connection with issues discussed in Sec. 1 we see that $r$ is directly related to distinct possibilities of productions of (i) Fermi balls or (ii) ribbon loops. For instance, for $r \approx 1$ the system seems to favor ribbon loops instead of Fermi balls. This same $r$ also controls the relation between the critical temperatures for formation of structureless domain walls $\left(T_{c}\right)$ and domain walls that support domain ribbons $\left(T_{\chi}^{c}\right)$. The specific relation is given by: $T_{\chi}^{c} / T_{c}=[3 /(5+r)]^{1 / 2}$.

The systems of two coupled real scalar fields introduced in Sec. 2 can be seen as the real bosonic sector of a supersymmetric theory [20]. Within this context, if we follow the point of view of supersymmetry to implement the idea of nesting domain ribbons inside domain walls, we can very naturally introduce fermions into the system. Thus supersymmetry may be very naturally used to guide investigations to more realistic models. For instance, instead of considering the $Z_{2} \times Z_{2}$ symmetry we may use the $Z_{2} \times U(1)$ symmetry, and this may make the domain wall charged, or yet the $U(1) \times U(1)$ symmetry that is the way to get to the string territory, where the original idea of introducing internal structure to topological defects was firstly implemented. Some investigations are connected to ideas presented in Sec. 11. Other investigations are directly related to the recent works [21, 22]. For 
instance, in 22] a $Z_{2} \times U(1)$ surface current-carrying domain wall model was investigated, but there the system is defined by a general potential of the form of our second system of two coupled fields, and all the results are implemented numerically due to difficulties in finding analytical solutions to the corresponding equations of motion. These works seem to deserve further considerations, now within the alternate way that considers systems defined via $H(\phi, \chi)$, because in this case there are interesting general situations where we can find explicit analytical solutions [20] to the equations of motion. The several motivations [22] for calculating internal quantities to such domain walls broaden with the fact that they may be calculated analytically. These and other related issues are presently under consideration.

We would like to thank J.R. Morris and R.F. Ribeiro for interesting comments. DB and FAB also thank Conselho Nacional de Desenvolvimento Científico e Tecnológico, CNPq, and Coordenação de Apoio ao Pessoal do Ensino Superior, CAPES, for partial suport and for a fellowship, respectively.

\section{References}

[1] A. Vilenkin and E.P.S. Shellard, Cosmic Strings and other Topological Defects (Cambridge University Press, Cambridge/UK, 1994).

[2] E. Witten, Nucl. Phys. B 249, 557 (1985).

[3] G. Lazarides and Q. Shafi, Phys. Lett. B 159, 261 (1985).

[4] R. MacKenzie, Nucl. Phys. B 303, 149 (1988).

[5] J.R. Morris, Phys. Rev. D 49, 1105 (1994).

[6] J.R. Morris, Phys. Rev. D 51, 697 (1995).

[7] J.R. Morris, Phys. Rev. D 52, 1096 (1995).

[8] D. Bazeia, R.F. Ribeiro, and M.M. Santos, Phys. Rev. D 54, 1852 (1996).

[9] D. Bazeia, M.J. dos Santos, and R.F. Ribeiro, Phys. Lett. A 208, 84 (1995). 
[10] D. Bazeia and M.M. Santos, Phys. Lett. A 217, 28 (1996).

[11] D. Bazeia, R.F. Ribeiro, and M.M. Santos, Phys. Rev. E 54, 2943 (1996).

[12] J.R. Morris and D. Bazeia, Phys. Rev. D 54 , 5217 (1996).

[13] A.L. MacPherson and B.A. Campbell, Phys. Lett. B 347, 205 (1995).

[14] J.R. Morris, Nested Domain Defects. To appear in Int. J. Mod. Phys. A; hep-ph/9707519.

[15] R. Jackiw, Phys. Rev. D 9, 1686 (1974).

[16] L. Dolan and R. Jackiw, Phys. Rev. D 9, 3320 (1974).

[17] S. Weinberg, Phys. Rev. D 9, 3357 (1974).

[18] R. Rajaraman, Solitons and Instantons (North-Holland, Amsterdam, 1982).

[19] P.M. Morse and H. Feshbach, Methods of Mathematical Physics (McGraw-Hill, New York, 1953) p. 1650.

[20] D. Bazeia and J.R. Morris, Domain walls and nontopological solitons in systems of two fields. Submitted to Int. J. Mod. Phys. A.

[21] P. Peter, Phys. Rev. D 46, 3335 (1992).

[22] P. Peter, J. Phys. A 29, 5125 (1996). 